\title{
Purcell-Enhanced Single-Photon Emission from Nitrogen-Vacancy Centers Coupled to a Tunable Microcavity
}

\author{
Hanno Kaupp, ${ }^{1,2}$ Thomas Hümmer, ${ }^{1,2}$ Matthias Mader, ${ }^{1,2}$ Benedikt Schlederer, ${ }^{1}$ Julia Benedikter, ${ }^{1,2}$ Philip Haeusser, ${ }^{1}$ \\ Huan-Cheng Chang, ${ }^{3}$ Helmut Fedder, ${ }^{4}$ Theodor W. Hänsch, ${ }^{1,2}$ and David Hunger ${ }^{1,2, *}$ \\ ${ }^{1}$ Fakultät für Physik, Ludwig-Maximilians-Universität, Schellingstraße 4, 80799 München, Germany \\ ${ }^{2}$ Max-Planck-Institut für Quantenoptik, Hans-Kopfermann-Straße 1, 85748 Garching, Germany \\ ${ }^{3}$ Institute of Atomic and Molecular Sciences, Academia Sinica, Taipei 106, Taiwan \\ 4. Physikalisches Institut, Universität Stuttgart, Pfaffenwaldring 57, 70569 Stuttgart, Germany \\ (Received 9 May 2016; revised manuscript received 3 September 2016; published 22 November 2016)
}

\begin{abstract}
Optical microcavities are a powerful tool for enhancing the fluorescence of individual quantum emitters. However, the broad emission spectra encountered in the solid state at room temperature limit the influence of a cavity, calling for an ultrasmall mode volume. We demonstrate Purcell-enhanced single-photon emission from nitrogen-vacancy centers in nanodiamonds coupled to a tunable fiber-based microcavity with a mode volume down to $1.0 \lambda^{3}$. We record cavity-enhanced fluorescence images and study several single emitters with one cavity. The Purcell effect is evidenced by enhanced fluorescence collection and tunable lifetime modification, and we infer an effective Purcell factor of up to 2. Furthermore, we show an alternative regime for light confinement, where a Fabry-Perot mode is combined with additional mode confinement by the nanocrystal itself. Simulations predict effective Purcell factors of up to 11 for nitrogenvacancy centers and 63 for silicon-vacancy centers, holding promise for bright single-photon sources and efficient spin readout under ambient conditions.
\end{abstract}

DOI: 10.1103/PhysRevApplied.6.054010

\section{INTRODUCTION}

Solid-state-based quantum emitters such as the nitrogenvacancy $(\mathrm{NV})$ center in diamond are promising for efficient single-photon sources [1,2], quantum memories [3-5], and quantum sensors [6-8], with functionality preserved also under ambient conditions. One of the central challenges is to efficiently access the quantum properties of the emitter by optical means. Optical microcavities [9] provide a powerful tool in this context since they enable the enhancement of light-matter interactions, and they offer an increase in spontaneous emission by the Purcell factor $C_{\text {eff }}=\left[3(\lambda / n)^{3} / 4 \pi^{2}\right]\left(Q_{\text {eff }} / V\right)$, together with the potential for near-unity collection efficiency $\beta=C_{\text {eff }} /\left(C_{\text {eff }}+1\right)$. Here, $V$ is the mode volume of the cavity, $n$ is the refractive index, and $Q_{\text {eff }}=\left(Q_{c}^{-1}+Q_{\mathrm{em}}^{-1}\right)^{-1}$ is the effective quality factor combining the quality factor of the cavity $\left(Q_{c}\right)$ and of the emitter $\left(Q_{\mathrm{em}}\right)[10,11]$.

For broadband emitters such as the $\mathrm{NV}$ center, $Q_{\mathrm{em}}$ as estimated from the linewidth of the emission spectrum is small at room temperature and limits the influence of a high $Q_{c}$, and cavities with an ultrasmall mode volume are required. Significant effort has been put into realizing

*david.hunger@physik.lmu.de

Published by the American Physical Society under the terms of the Creative Commons Attribution 3.0 License. Further distribution of this work must maintain attribution to the author(s) and the published article's title, journal citation, and DOI. coupled systems with both bulk micro- and nanocavities [12-21], as well as tunable open-access microcavities [22-26]. While high-quality membranes hosting unperturbed $\mathrm{NV}$ centers currently appear as a favorable solution for cavity-quantum-electrodynamics experiments at cryogenic temperature [15,20,25], nanodiamonds remain an interesting choice [27] for coupling to cavities to enhance signals for room-temperature applications such as magnetometry [6-8] or single-photon sources [22,24]. However, the required smallest mode volumes have remained challenging to achieve for tunable microcavities [28] and are mostly demonstrated in bulk cavities, where finding or placing a single emitter in the cavity-field maximum is difficult.

Here, we realize an open-access tunable microcavity $[25,28-34]$ with a mode volume as small as $1 \lambda^{3}$, and we use it to demonstrate Purcell enhancement of the emission of $\mathrm{NV}$ centers in nanodiamonds. The tunable cavity lends itself to cavity-enhanced fluorescence imaging of large areas of the sample by scanning-cavity microscopy $[30,35,36]$, allowing us to study several single emitters with one and the same cavity. Photon-collection rates from single-NV centers of up to $1.6 \times 10^{6} \mathrm{~s}^{-1}$ and a Purcell factor of 2 are observed. The large lifetime change gives the first evidence that an even smaller mode volume is achieved, through a combination of the Fabry-Perot mode with additional transversal mode confinement of a suitably sized nanodiamond that supports a waveguide mode. To support this picture, we perform measurements and numerical simulations with a planar Fabry-Perot cavity and study 
the lifetime as a function of the mirror separation. For an optimized geometry, the simulations predict an effective Purcell factor of up to 11 for NV centers, and an outcoupling efficiency from the cavity into a mode with a low numerical aperture (NA) of up to $76 \%$, limited by the intrinsic loss of the used mirror coating.

\section{ULTRASMALL-MODE-VOLUME TUNABLE CAVITY}

The microcavity is assembled from a planar mirror onto which the sample is applied and a concave mirror on the tip of an optical fiber [29]; see Fig. 1(a). The tip of the cavity fiber is shaped by advanced $\mathrm{CO}_{2}$-laser machining [37,38]. In a first step, the extent of the end facet is tapered to enable submicron mirror separations, which is crucial when aiming at the smallest mode volumes. Therefore, multiple laser pulses are applied in circular patterns to the edge of the end facet to crop the outer part of the fiber, resulting in a protruding plateau with a diameter that is typically below

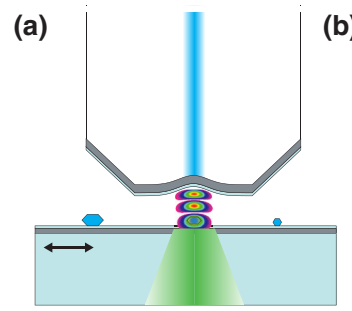

(c)

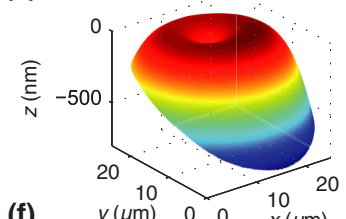

(f)
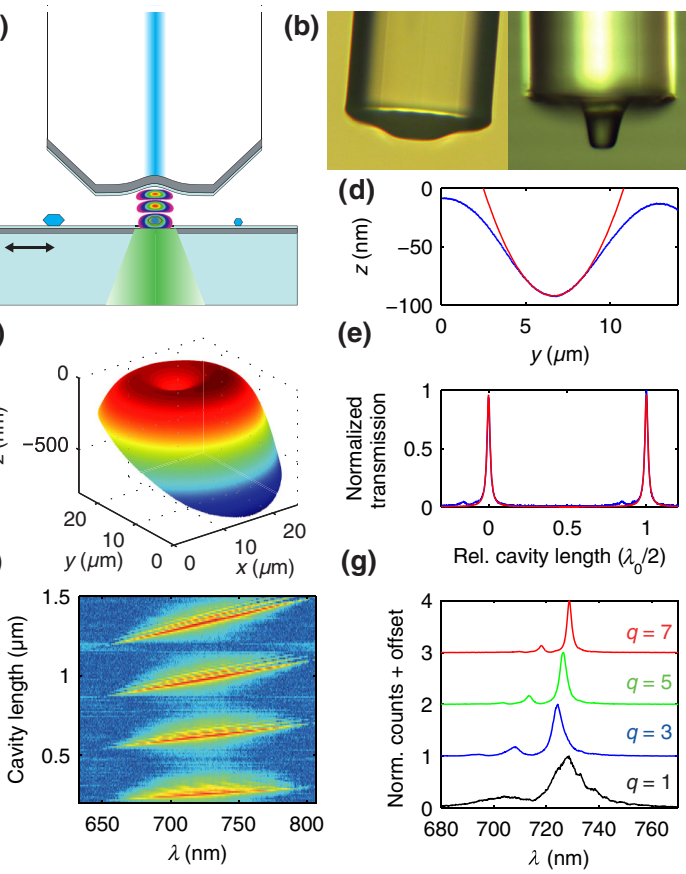

(g)

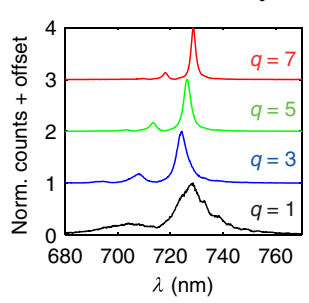

FIG. 1. (a) Sketch of the cavity consisting of a laser-machined and mirror-coated fiber and a macroscopic mirror carrying nanodiamonds with NV centers. The large mirror is mounted on a three-axis nanopositioning stage for spatial scanning, the cavity length is controlled by an additional piezoelectric actuator. (b) Microscope images of two laser-shaped fiber tips. (c) 3D profile of a laser-machined and silver-coated fiber tip. (d) Cut through the center of the structure shown in (c) (the blue curve) together with a parabolic fit (the red curve). (e) Cavity transmission probed with a narrow-band laser as a function of the cavity length. (f) Series of cavity transmission spectra under broadband illumination as a function of the cavity length, showing tunability for cavity lengths down to $\lambda_{0} / 2$. (g) Individual spectra from (f) for different mode orders $q$.
$20 \mu \mathrm{m}$. Figure 1(b) shows two examples of different shape. Next, a concave depression aligned with the fiber core is produced within the center of the plateau using a few weak laser pulses [39]. Figure 1(c) shows the topography of a machined fiber tip measured by white-light interferometry. The central part of the profile is well fitted by a parabola, yielding a radius of curvature $r_{c}=90 \mu \mathrm{m}$, while the full profile fits to a Gaussian function with $1 / e$ diameter $D=7 \mu \mathrm{m}$ and a structure depth $z<100 \mathrm{~nm}$ [Fig. 1(d)].

When choosing an optimal mirror coating, both the mirror reflectivity and the field penetration need to be considered. While dielectric coatings provide the highest reflectivity and low loss, they exhibit significant field penetration into the coating, which increases the mode volume. Optimizing the Purcell factor for broadband emitters shows that metal coatings with a few tens of nanometers penetration are advantageous, despite the higher loss $[28,40,41]$. We coat the fiber tip with $60 \mathrm{~nm}$ and the planar mirror with $33 \mathrm{~nm}$ of silver, both finished with a 20-nm glass capping to prevent oxidation. This procedure yields reflectivities of $R_{2}=96 \%$ and $R_{1}=88 \%$ and absorption loss of about $A=4 \%$, while scattering loss is found to be negligible. The two mirrors define an open and tunable plano-concave Fabry-Perot cavity that outcouples up to $\eta_{c}=0.51$ of the light through the planar mirror into the detection channel (see the Appendix). We measure a cavity finesse of $\mathcal{F}=42 \pm 1$ at $\lambda_{0}=690 \mathrm{~nm}$ by recording the cavity transmission of a narrow-band laser when tuning the cavity length over one free spectral range [Fig. 1(e)].

We determine the mode volume of the cavity by measuring the optical cavity length $d$ and the mode waist $w_{0}$, and we use the expression $V=\pi w_{0}^{2} d / 4$. To determine $d$, we record the cavity transmission under broadband illumination with a spectrometer-see Figs. 1(f) and 1(g) - and evaluate the separation and the location of the resonances. For example, from the change of the resonance wavelength $\Delta \lambda$ when changing the mirror separation by $\Delta z$, one can directly infer the mode order $q=2 \Delta z / \Delta \lambda$. In this way, we prove that the shortest resonant-cavity length $d=\lambda_{0} / 2$ is reached without touching the planar mirror with the fiber tip, and full tunability is ensured even for the fundamental resonance $q=1$. By scanning the cavity over a pointlike object such as a single-NV center and collecting the fluorescence emitted into the cavity [Figs. 2(b) and 2(c)], the mode waist can be inferred from the observed size of the point-spread function $w_{\text {det }}$ in a cavity-enhanced fluorescence image (see the Appendix). We determine a minimal $w_{0}=$ $1.1 \mu \mathrm{m}$ at $d=\lambda_{0} / 2$, such that a minimal cavity-mode volume $V=1.0 \lambda_{0}^{3}\left(0.34 \mu \mathrm{m}^{3}\right)$ is achieved.

\section{CAVITY-ENHANCED EMISSION FROM SINGLE-NV CENTERS}

We use the described cavity to study cavity-enhanced single-photon emission from individual NV centers. 

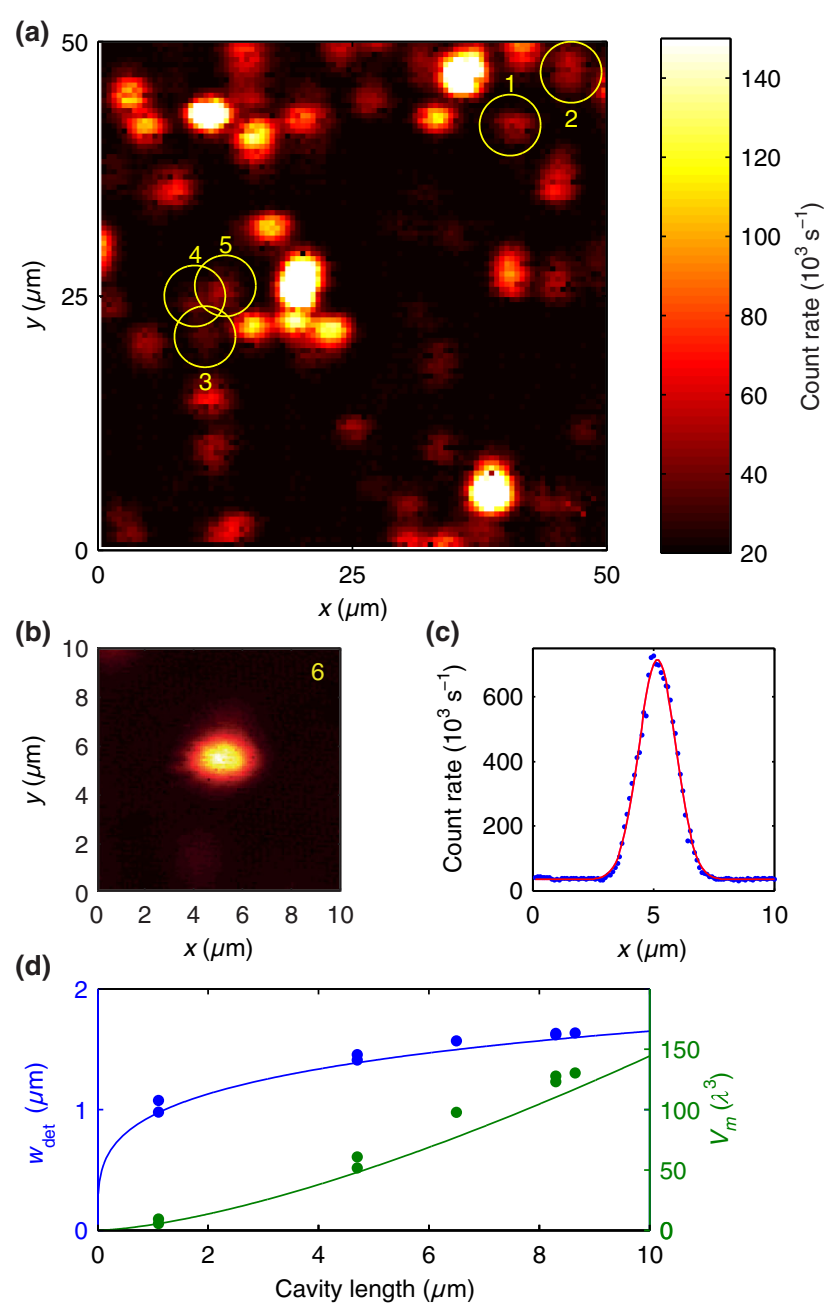

FIG. 2. (a) Cavity-enhanced flourescence image showing several single emitters (the circles). The cavity length is stabilized on resonance with the excitation light at a length of about $10 \mu \mathrm{m}$ while scanned laterally. (b) Cavity scan of a single-NV center at $1.1-\mu \mathrm{m}$ cavity length. (c) Vertical cut through the scan in (b) together with a Gaussian fit, yielding $w_{\text {det }}=1.1 \mu \mathrm{m}$. (d) Measured size of the point-spread function (the blue points) as a function of the cavity length, together with the value calculated from the radius of curvature of the fiber mirror (the blue line). Mode volume calculated from the measured $w_{\text {det }}$ (the green points), together with the expected value (the green line).

The nanodiamond sample (Van Moppes) has a size distribution between 100 and $200 \mathrm{~nm}$, where a reasonable fraction of the crystals contain individual NV centers. The large crystal size is chosen to support mode confinement by the crystal within a cavity with $\lambda / 2$ mirror separation. The sample is directly spin coated or drop cast onto the planar mirror. As a first step, we record a cavity-enhanced fluorescence image of a large area of the sample by scanning the planar mirror, while the cavity length is actively stabilized to a mirror separation of around $10 \mu \mathrm{m}$. At this distance, the excitation light and the NV emission spectrum are simultaneously resonant with different cavity modes. Figure 2(a) shows a scan area of $(50 \mu \mathrm{m})^{2}$, where about 50 emitters are visible. The excitation power is $3.6 \mathrm{~mW}$ at $532 \mathrm{~nm}$. The second-order correlation function $g^{(2)}(\tau)$, as well as the saturation behavior for the five marked emitters, is recorded. A closer view on another, more isolated single-NV center (NV6) is shown in Figs. 2(b) and 2(c), where the cavity length is stabilized at $d=1.1 \mu \mathrm{m}$. We observe a spatial resolution of $1.1 \mu \mathrm{m}$, a high peak-count rate $\left(>6 \times 10^{5} \mathrm{~s}^{-1}\right)$, and clear antibunching with $g^{(2)}(0)=0.21$. The background fluorescence next to the crystal is more than a factor of 20 lower, indicating the potential for clean single-photon emission. The obtained value for $g^{(2)}(0)$, however, suggests an additional background stemming from the nanocrystal in this case. With the simultaneous excitation and fluorescence enhancement and the emission into the well-collectable cavity mode, the cavity enables enhanced count rates and provides spatial and spectral filtering at the same time. Cavity-enhanced scanning fluorescence microscopy thus promises net signal improvement compared to confocal microscopy.

As an example for the single-emitter fluorescence obtainable from the cavity, we discuss the results for one NV center (NV1) in more detail. To identify the mirror separation for optimal single-photon production, we record the count rate and the $g^{(2)}$ function for various mirror separations. We find the highest signal-to-background ratio at $d=1.1 \mu \mathrm{m}$, which is the shortest mirror separation for which the cavity is simultaneously resonant for excitation $(q=4)$ and fluorescence emission $(q=3)$. We thus use it for the following count-rate and $g^{(2)}$ measurements. We measure the $g^{(2)}$ function at low excitation power and fit it with the function $g^{(2)}(\tau)=1+p\left(b e^{-|\tau| / \tau_{2}}-(1+b) e^{-|\tau| / \tau_{1}}\right)$, which includes antibunching, bunching, and background; see Fig. 3(a). The fit value of $g^{(2)}(0)=0.27$ proves that it is a single emitter. Figure 3(b) shows a measurement of the intensity-dependent fluorescence rate, which is fitted well by a saturation part and a linear contribution accounting for the background: $K=K_{\infty} I /\left(I_{\text {sat }}+I\right)+a I$ (see the Appendix). We find a saturation count rate $K_{\infty}=6.9 \times 10^{5} \mathrm{~W} \mathrm{~m}^{-2}$, a saturation intensity $I_{\text {sat }}=$ $0.49 \times 10^{9} \mathrm{~W} \mathrm{~m}^{-2}$ and a linear background parameter $a=1.0 \times 10^{4} \mathrm{~s}^{-1} / 10^{9} \mathrm{~W} \mathrm{~m}^{-2}$. The background count rate recorded next to the emitters yields the same value for $a$; i.e., the diamond crystal itself does not contribute any notable background in this case. The reported count rates are raw values as detected with avalanche photodiodes (APDs). Accounting for the detection efficiency of the optical setup of $43 \%$ (see the Appendix), we find that $1.6 \times 10^{6}$ photons per second are collected by the first lens.

To quantify the enhancement of the count rate by the cavity, we study several emitters in the cavity and compare the results with confocal measurements $(\mathrm{NA}=0.75)$ of NV centers on a glass substrate. We record the $g^{(2)}$ function and the saturation count rate of each emitter and compare 
(a)

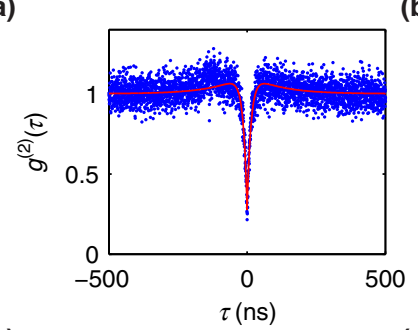

(c)

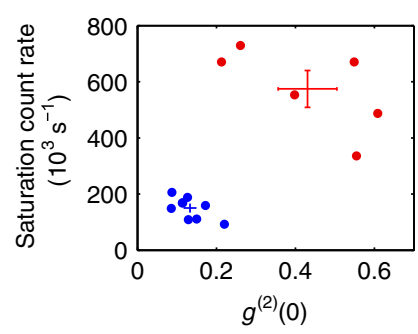

(b)

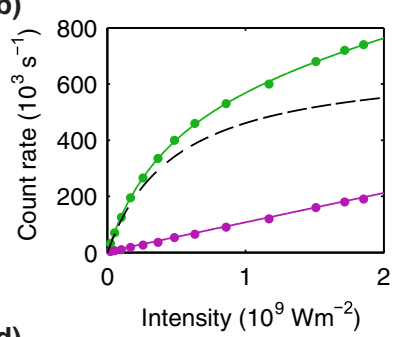

(d)

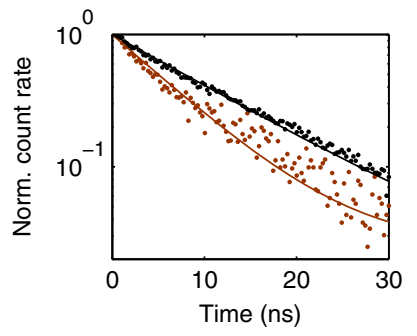

FIG. 3. (a) Autocorrelation measurement for NV1 yielding $g^{(2)}(0)=0.27$ without background subtraction. (b) Saturation measurement (the green dots) with a fit of the same NV center. Background fluorescence (the purple dots) with a linear fit. $\mathrm{NV}$-center fluorescence subtracted by the background (the black dashed line). (c) Comparison of the saturation count rates and single-photon purities for NV centers on glass (the blue dots) and inside the cavity at $d=1.1 \mu \mathrm{m}$ (the red dots). An average enhancement by a factor of 3.8 is found. (d) Lifetime measurement of NV1 at $d=2 \mu \mathrm{m}$ (black) and $d=\lambda_{0} / 2$ (brown) with monoexponential fits.

the results by plotting the saturation count rate versus $g^{(2)}(0)$ for cavity and free-space emission; see Fig. 3(c). Taking background fluorescence into account, we expect all NVs in the cavity with $g^{(2)}(0) \lesssim 0.7$ to be single emitters. The evaluation yields an average detected saturation count rate per $\mathrm{NV}$ center of $K_{\infty}^{c}=5.7 \times 10^{5} \mathrm{~s}^{-1}$ inside the cavity, in comparison to $K_{\infty}=1.5 \times 10^{5} \mathrm{~s}^{-1}$ on the glass substrate. This value corresponds to an average observed enhancement factor of 3.8.

To study the effect of the cavity on the excited-state lifetime, we perform time-correlated single-photon counting under pulsed excitation at different mirror separations. While no significant lifetime dependence on the mirror separation is observed when $d>0.8 \mu \mathrm{m}$, a notable lifetime reduction is present when $d=\lambda_{0} / 2$. For NV1, we find $\tau=11 \pm 0.5 \mathrm{~ns}$ when $d=2 \mu \mathrm{m}$ and $\tau_{c}=7 \pm 1.5 \mathrm{~ns}$ when $d=\lambda_{0} / 2$; see Fig. 3(d). The large uncertainty of the value at $d=\lambda_{0} / 2$ stems from a low signal-tobackground ratio since the excitation light is off resonant with the cavity at this mirror separation. The background yields a nonmonoexponential decay, which we consider as error bars by testing different fit domains. We note that this measurement yields a comparison between a "half cavity" with one mirror for large $d$ and a full cavity at small $d$, such that a constant effect on the lifetime is always present due to the sample mirror. We estimate the corresponding lifetime

change from a statistical comparison of lifetimes both on the mirror and on a glass substrate (reduction by a factor of $1.3 \pm 0.3$ ), as well as from corresponding finite-differencetime-domain (FDTD) simulations averaged over dipole orientations (reduction factor 1.8), suggesting a free-space lifetime of up to $\tau_{0}=20 \mathrm{~ns}$. With this estimate, we obtain a value for the effective Purcell factor of $C_{\text {eff }}=\left[\left(\tau_{0} / \tau_{c}\right)-1\right] /$ $\mathrm{QE}=2 \pm 0.6$ when assuming a quantum efficiency $\mathrm{QE}=1$, and correspondingly larger values for $\mathrm{QE}<1$ [42] (see the Appendix).

We estimate the theoretically expected count-rate enhancement and Purcell factor as follows: The photon rate coupled out of the cavity under saturation conditions is given by $K_{\infty}^{c}=C_{\text {eff }} \gamma_{\mathrm{r}} \eta_{c}$, where $\gamma_{\mathrm{r}}$ is the radiative-decay rate (see the Appendix). For the experimental parameters used in the measurements described above, we find $C_{\text {eff }}=$ 0.12 for $V=5 \lambda_{0}^{3}, Q_{c}=126$, and $Q_{\mathrm{em}}=\left(\lambda_{0} / \Delta \lambda\right)=8$, calculated from the center wavelength $\lambda_{0}=690 \mathrm{~nm}$ and the FWHM $\Delta \lambda=90 \mathrm{~nm}$ of the emission spectrum. The fraction of photons leaving the cavity through the outcoupling mirror is $\eta_{c}=0.51$, and the cavity mode is fully collected by the objective. For the case of NV centers on a glass substrate, the dipole-radiation pattern is affected by the air-glass interface [43,44]. For the given NA, we calculate a collection efficiency of $\eta_{\Omega}=0.16$ and a photon-collection rate $K_{\infty}=\gamma_{\mathrm{r}} \eta_{\Omega}$. The detection efficiency after the objective is assumed to be equal to the cavity case. The total expected enhancement is then $K_{\infty}^{c} / K_{\infty}=$ $C_{\text {eff }}\left(\eta_{c} / \eta_{\Omega}\right)=0.38$, in contrast to the value found in the experiment.

For the lifetime measurement at $d=\lambda_{0} / 2$, we calculate $C_{\text {eff }}=0.6$ for $V=1 \lambda_{0}^{3}, Q_{c}=42, Q_{\mathrm{em}}=8$, again significantly smaller than the value found in the experiment.

This result shows that the simplified treatment does not properly describe the situation. In fact, the applied formula is derived in the limit where the cavity mirrors subtend a negligible solid angle and where the dipole remains far from surfaces. For a more accurate treatment, we perform analytical $[43,44]$ and FDTD simulations (see the Appendix).

We identify three aspects that influence the spontaneous emission beyond the simple treatment. (i) The proximity between the metal mirror and the sample leads to selfinterference of the dipole radiation over a large angular range as well as to some amount of near-field coupling $[43,44]$. A FDTD simulation for an orientation-averaged dipole located in a nanodiamond on a metal mirror predicts a lifetime reduction factor of 1.8 compared to the situation without the mirror, as well as negligible nonradiative decay $(<10 \%)$. (ii) The large solid angle subtended by the cavity mirrors significantly affects the mode structure beyond the fundamental cavity mode and consequently influences (e.g., inhibits) the decay into modes other than the fundamental cavity mode $[45,46]$. (iii) For a suitable crystal size and the smallest mirror separation, the nanodiamond provides additional lateral-mode confinement (see below). 
All of these aspects are captured by FDTD simulations of the emitter inside the cavity, and we obtain an effective Purcell factor of $C_{\text {eff }}=1.4$ at $d=1.1 \mu \mathrm{m}$, with a corresponding count-rate enhancement $K_{\infty}^{c} / K_{\infty}=$ $C_{\text {eff }} \eta_{c} / \eta_{\Omega}=4.5$, and $C_{\text {eff }}=11$ at $d=\lambda_{0} / 2$. While the predicted count-rate enhancement compares well with the experimental value, the simulation predicts a significantly larger lifetime reduction for the shortest mirror separation. We show below that this large reduction results from the mode confinement caused by the nanocrystal.

Expected contributions to the deviation from the experimental results are a reduced coupling strength due to nonideal dipole orientation and an emitter location within the nanodiamond away from the cavity-field maximum. The observed lifetime change is, furthermore, influenced by a finite quantum efficiency, and, e.g., a QE value of 0.2 would resolve the discrepancy.

\section{WAVEGUIDE EFFECT}

In the following, we present measurements and simulations that support the picture that significantly larger Purcell enhancement can be achieved by combining a Fabry-Perot mode with additional transversal mode confinement of a suitably sized nanodiamond that supports a waveguide mode. To separate the waveguide effect from the transversal mode confinement due to the cavity mirrors, we study a planar Fabry-Perot geometry. In the experiment, we use a cavity fiber with a cleaved planar end facet, whose edges are mechanically polished off to allow for the smallest mirror separations. The fiber has an identical coating as in the previous case, while the large mirror has higher transmission due to a thicker glass capping $(60 \mathrm{~nm})$, resulting in $\mathcal{F}=28$ and $\eta_{c}=0.64$ at $700 \mathrm{~nm}$. We study ensembles of NV centers with bright fluorescence and a negligible background to avoid the influence from a distance-dependent background on the lifetime [47]. The nanodiamonds with a diameter distribution peaking between 100 and $150 \mathrm{~nm}$ are irradiated with $\mathrm{He}$ ions to achieve a high concentration of NV centers (approximately $10^{2}$ per crystal) $[48,49]$.

We measure the fluorescence lifetime of the NV-center ensemble for different mirror separations [50], where we tune the mirror separation with a piezoelectric actuator and calibrate the optical cavity length $d$ as well as the geometrical mirror separation $d_{0}$ from fluorescence spectra [51]. Each decay trace is fitted with a monoexponential decay, from which we obtain the lifetime $\tau\left(d_{0}\right)$ [52]. Figure 4(a) shows a sample measurement, and Fig. 4(b) presents individual traces for the mirror separations indicated in Fig. 4(a). For cavity lengths below $2 \mu \mathrm{m}$, the lifetime is modulated noticeably.

Whenever the cavity is resonant (off resonant) with the emission spectrum, the lifetime is reduced (increased) due to the variation of the local density of states. The shortest (longest) detected lifetime of $\tau_{c}=11.2 \mathrm{~ns}$ (23.7 ns) corresponds to a lifetime reduction (enlargement) of $40 \%$ (25\%) compared to the lifetime of $\tau_{m}=18.9 \mathrm{~ns}$ obtained (a)

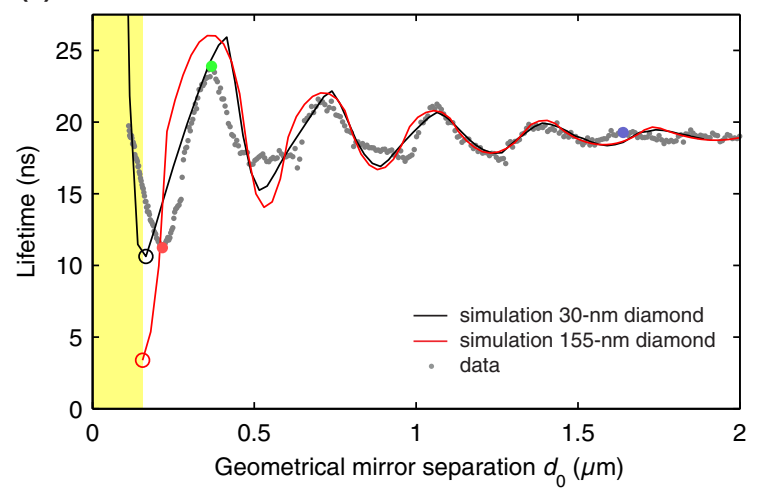

(b)

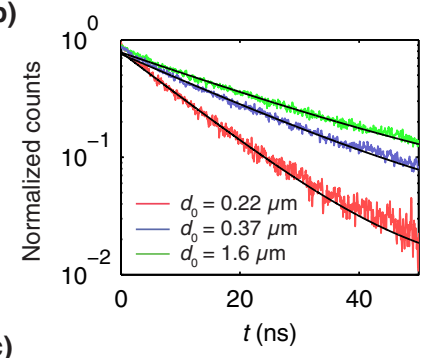

(c)

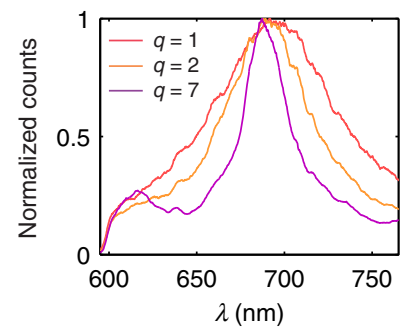

(d)
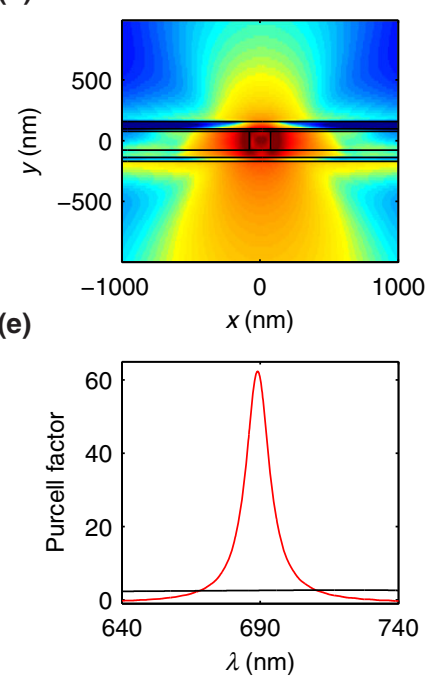

FIG. 4. (a) Measured lifetime $\tau\left(d_{0}\right)$ as a function of the mirror separation (gray dots) together with FDTD simulations for a diamond cube of edge length $30 \mathrm{~nm}$ (black line) and $155 \mathrm{~nm}$ (red line). The filled colored dots indicate the positions at which the data sets shown in (b) are taken. The yellow area shows the distance range where the fiber touches the large diamond. (b) Lifetime traces for different cavity lengths with fits (black), (red: $\tau=11.2 \mathrm{~ns}$, blue: $19.3 \mathrm{~ns}$, green: $23.9 \mathrm{~ns}$ ). (c) Cavity emission spectra at resonances corresponding to longitudinal mode orders $q=1,2,7$. (d) Simulation of the intensity distribution of a dipole located at the center (logarithmic scale, red: high, blue: low). The intensity is confined between the two silver mirrors (horizontal black lines, including the spacer layers) and localized to the nanocrystal (black square). Outcoupling occurs predominantly through the thinner bottom mirror. (e) Simulated Purcell factor as a function of the wavelength for a $155 \mathrm{~nm}$ (red, corresponding to open circle in (a)) and a $30 \mathrm{~nm}$ (black) diamond. 
when the cavity fiber is not present. Together with the effect of the sample mirror alone, this result suggests a free-space lifetime of up to $\tau_{0}=34 \mathrm{~ns}$. With this knowledge, we obtain an effective Purcell factor of $C_{\text {eff }}=\left[\left(\tau_{0} / \tau_{c}\right)-1\right] /$ $\mathrm{QE}=2.0$ for $\mathrm{QE}=1$.

We study several nanocrystals in a similar manner and observe a shortest lifetime of $\tau_{c}=6.7 \mathrm{~ns}$ with comparable relative lifetime changes. At the same time, the integrated fluorescence increases by up to a factor of 40 from $d=2 \mu \mathrm{m}$ to $d=\lambda / 2$. This increase results from the fact that the cavity has a negligible effect for large mirror separations and only the emission transmitted through the planar mirror $\left(T_{1}=0.11\right)$ is collected with a small collection efficiency of $\eta_{\Omega}=0.16$. By contrast, for short cavities, the Purcell effect leads to a dominant emission into the cavity mode, a fraction $\eta_{c}=0.64$ of which is coupled out to the detector side and is entirely collected by the objective (see the Appendix).

The fluorescence is spectrally filtered by the cavity, yielding a resonance with a linewidth depending inversely on the mirror separation; see Fig. 4(c). Since the planar cavity has an angle-dependent resonance wavelength and we are collecting a large angular range, the spectral width of the resonance is, however, not directly indicative of the quality factor.

We compare the measurements to FDTD simulations (see the Appendix) and study a dipole in a diamond cube with edge lengths of 155 and $30 \mathrm{~nm}$ for comparison. Figure 4(d) shows a cross section of the geometry and the intensity distribution of a dipole oriented along the $x$ axis located in the center. A strong confinement of the intensity to the crystal is visible (note the logarithmic color scale), as well as the directional outcoupling through the bottom mirror. The outcoupled mode has a NA of 0.26 (0.38 after refraction at a glass-air interface), which can be easily collected by an objective.

For each cavity length, the simulation evaluates the Purcell factor as a function of the wavelength $C(\lambda)$. Figure 4(d) shows an example for the 155-nm crystal at $d=\lambda / 2$, yielding a peak Purcell factor of $C=63$ compared to the nanocrystal in vacuum. To enable a comparison with the experimental data, we normalize $C(\lambda)$ to the Purcell factor $C_{m}(\lambda)$ obtained for simulations where the fiber mirror is removed, and we average over the NV emission spectrum $S(\lambda)$.

The expected lifetime change is then determined to be $\tau_{m} / \tau_{c}=\int_{-\infty}^{\infty}(C(\lambda)+1)\left(C_{m}(\lambda)+1\right)^{-1} S(\lambda) d \lambda /$ $\int_{-\infty}^{\infty} S(\lambda) d \lambda$. The simulations for the parallel dipole are in good agreement with the measurements. Contributions of the normal dipole component are expected to play a negligible role since this orientation is only weakly excited and coupled to the cavity. For the $155-\mathrm{nm}$ diamond, a maximal effective Purcell factor of $C_{\text {eff }}=11$ is predicted when the fiber touches the diamond.

In contrast, the $30-\mathrm{nm}$ diamond yields a much smaller Purcell factor, and the enhancement spectrum $C(\lambda)$ does not show a clear resonance [see Fig. 4(d)]. We note that, for the ideal situation of a dipole between two lossless planar mirrors without the presence of a nanocrystal, an analytical calculation predicts a maximal Purcell factor of $C=2$ [45], which is close to the value found for the $30-\mathrm{nm}$ crystal.

Some intuition about the confinement effect can be drawn from modeling the crystal as a cylindrical waveguide [53] with refractive index $n_{d}=2.4$ and solving the Helmholtz equation for the electric field $\left(\nabla^{2}+k^{2}\right) E=0$ in one dimension. Here, $k=2 \pi n_{r} / \lambda$, with $n_{r}=n_{d}$ for $|r| \leq$ $b$ and 1 for $|r|>b, r$ is the transverse coordinate, and $b$ the waveguide radius.

We find that the propagating waveguide mode shows the strongest confinement around $b=70 \mathrm{~nm}$, yielding a minimal effective mode radius $\left(1 / e^{2}\right)$ of $w_{0}=160 \mathrm{~nm}$. A segment of such a nanowire can now be considered to be introduced into a planar Fabry-Perot cavity with mirror separation $d=\lambda / 2 n_{\text {eff }}$, where $n_{\text {eff }}$ is the effective refractive index experienced by the propagating mode (we find $n_{\text {eff }}=$ 1.88 for $b=70 \mathrm{~nm}$ ). The mode volume of such a cavity would amount to $V=\pi w_{0}^{2} \lambda / 8 n_{\text {eff }}=0.07\left(\lambda / n_{\text {eff }}\right)^{3}$, substantially smaller than what is achievable by conventional curved-mirror Fabry-Perot cavities.

Together with an effective quality factor $Q_{\text {eff }}=8$, this estimation yields $C_{\text {eff }}=8$, in reasonable agreement with the FDTD simulation. From the more reliable FDTD simulations, we find an optimal crystal size of around $155 \mathrm{~nm}$ for a cubic shape. $\mathrm{A} \pm 10 \%$ size variation does not significantly affect the maximal $C$, while the enhancement rapidly diminishes for crystals $<140 \mathrm{~nm}$, and no additional enhancement remains for $30 \mathrm{~nm}$.

The measurement also suggests the presence of a contribution of the waveguide effect. Experimental imperfections, averaging over dipole orientation, and nonideal quantum efficiency are expected to lead to a reduced lifetime modification. Also, the increase of the measured lifetime towards the smallest $d$ indicates a crystal size below $140 \mathrm{~nm}$, for which simulations predict a smaller lifetime change since the waveguide mode is not fully supported. The imperfections are apparent, e.g., at the lifetime minima corresponding to the resonances $q=2$ and $q=3$, where the lifetime reduction in the data remains smaller than in the simulation for both crystal sizes [54]. However, for $q=1$, where the waveguide effect is expected to be present, the experimental variation is as large as the simulated ideal variation for the 30 -nm crystal. Consequently, the Purcell factor in the experiment needs to be larger than in the simulation to compensate for the imperfections.

\section{CONCLUSION}

We demonstrate that improved laser machining enables the realization of stable Fabry-Perot cavities with mode volumes down to $1 \lambda^{3}$, while maintaining full tunability. Multiple emitters can be investigated with a single cavity, 
and efficient single-photon extraction is possible even for broadband emitters. The Purcell enhancement, together with high outcoupling efficiency into a well-collectable mode, allows net count rates exceeding free-space collection.

Furthermore, we have shown that suitably chosen nanodiamonds can provide exceptional mode confinement by a waveguide effect. Predicted ideal Purcell factors up to 63 could be fully exploited with narrow-band emitters such as the silicon-vacancy $(\mathrm{SiV})$ center in diamond, and effective Purcell factors for NV centers up to 11 are expected. Such high Purcell factors would lead to high efficiencies $\beta=0.98$ and 0.91 for SiV and NV centers, respectively, and at the same time bring background fluorescence to a negligible level.

In an optimized setting, source efficiencies of $\beta \eta_{c}=$ $82 \%$ for $\mathrm{SiV}$ centers and $76 \%$ for NV centers can be achieved, limited by the finite absorption loss in the metal mirrors. While our experiments already indicate a contribution due to the waveguide effect, we expect that more controllably fabricated diamond nanostructures $[8,55,56]$ can unfold the full potential of this approach and pave the way for ultrabright single-photon sources and superior readout of single spins under ambient conditions.

\section{ACKNOWLEDGMENTS}

We thank Aniket Agrawal and Tolga Bagci for their contributions to the experiment, as well as Philipp Altpeter for his assistance in the clean room. Fruitful discussions with Christoph Becher, Jason Smith, and Jörg Wrachtrup are acknowledged. This work has been funded by the European Union 7th Framework Program under Grant Agreement No. 61807 [57] and the DFG Cluster of Excellence NIM. T.W. H. acknowledges funding from the Max-Planck Foundation.

\section{APPENDIX: METHODS}

\section{Mirror coating}

The fiber tips are coated with a 60-nm silver film and finished with a 20-nm glass capping to prevent oxidation. Consistent with simulations [58], we measure a reflectivity of $R_{2}=(92 \pm 2) \%$ at $532 \mathrm{~nm}$. The macroscopic planar mirror is prepared on a low-autofluorescence glass substrate with a 33-nm-thick silver layer and an analogous glass capping. At a wavelength of $532 \mathrm{~nm}$, we measure a mirror transmission of $T_{1}=15 \%$. A simulation yields the transmission and absorption loss of the two mirrors at $700 \mathrm{~nm}: T_{1}=8 \%, T_{2}=0.8 \%$ and $A_{1}=4 \%, A_{2}=3 \%$.

With atomic-force-microscopy measurements, we determine the surface roughness of the silver mirrors to be $<5 \mathrm{~nm}$ rms, such that scattering loss plays a negligible role $(<0.5 \%)$. The measured finesse agrees well with the transmission and loss values, and we calculate the expected fraction of photons leaving the cavity through the macroscopic mirror to be $\eta_{c}=T_{1} /\left(T_{1}+T_{2}+A_{1}+A_{2}\right)=0.51$.

Despite the lossy character of the metal coatings, a high outcoupling efficiency is achieved. For the plane-plane cavity, the glass-capping layer of the macroscopic mirror is increased to $60 \mathrm{~nm}$, resulting in an increased transmission, $T_{1}=24 \%$ at $532 \mathrm{~nm}$ and $T_{1}=11 \%$ at $700 \mathrm{~nm}$, respectively. The outcoupling efficiency amounts to $\eta_{c}=0.64$ at $700 \mathrm{~nm}$. For a mirror coating that optimizes the overall source efficiency for the waveguide scenario, we find that $\eta_{c} C /(C+1)=0.83$ can be achieved.

\section{Mode volume}

The mode volume of a plano-concave cavity is given by $V=\pi w_{0}^{2} d / 4$, with the mode waist $w_{0}^{2}=(\lambda / \pi) \sqrt{r_{c} d-d^{2}}$ and the optical cavity length $d=(\lambda / 2)[q+(\zeta / \pi)] \approx$ $q(\lambda / 2)$ for $r_{c} \gg d$. Here, $q$ is the longitudinal-mode order, and $\zeta=\arccos \sqrt{1-d / r_{c}}$ is the Gouy phase. The finite conductivity of the silver mirror and the capping layer leads to some field penetration into the mirror, such that the geometrical mirror separation $d_{0}=(\lambda / 2)\{q+[(\zeta-\phi) / \pi]\}$ is smaller than $d$. This circumstance is accounted for by the average deviation of the reflection phase from $\pi, \phi=$ $\pi-\left(\phi_{1}+\phi_{2}\right) / 2$. At $\lambda=700 \mathrm{~nm}, \phi_{1} \approx \phi_{2}=0.72 \pi$ for our mirrors [59], and we calculate an air gap of $d_{0}=$ $260 \mathrm{~nm}$ for $q=1$. This value reduces further when a nanodiamond of significant size is placed in the cavity mode due to the effective refractive-index change.

\section{Point-spread function}

In cavity-enhanced fluorescence scans, the observed point-spread function is the product of the cavity mode for emission $\left(w_{0}\right)$ and excitation $\left(w_{\mathrm{e}}=\sqrt{\lambda_{\mathrm{e}} / \lambda} w_{0}\right)$. The size of the point-spread function is then $w_{\text {det }}=$ $w_{\mathrm{e}} w_{0} / \sqrt{w_{\mathrm{e}}^{2}+w_{0}^{2}}$, which can be used to determine $w_{0}$. The experimental values agree well with a calculation for different cavity lengths when using the measured radius of curvature of the fiber mirror; see Fig. 2(d).

\section{Setup}

The cavity is embedded into a confocal-microscope setup, similar to the one described in Ref. [23]. Excitation is performed with either a cw laser at $532 \mathrm{~nm}$ or a band-pass-filtered supercontinuum source (of an approximately 50 -ps pulse length and $20-\mathrm{MHz}$ repetition rate) through a coverslip-corrected microscope objective $(\mathrm{NA}=0.75)$ through the planar mirror. Alternatively, the excitation can be performed through the cavity fiber. In this case, the distance-dependent mode matching between the fiber mode and the cavity mode needs to be accounted for, which varies from $93 \%$ at $d=10 \mu \mathrm{m}$ to $55 \%$ at $d=\lambda_{0} / 2$. For pulsed excitation, we use average excitation powers ranging from a couple dozen to a few hundred microwatts. The planar mirror is mounted on a three-axis slip-stick 
nanopositioner (Attocube ECS3030), allowing us to scan the mirror for suitable emitters. The fiber is mounted on a stacked piezoelectric actuator which allows for precise tuning and the stabilization of the cavity length. The latter is performed by using the green excitation light transmitted through the cavity to generate a cavity-length-dependent feedback signal, which is fed to the stacked piezoelectric actuator controlling the fiber position.

\section{Excitation intensity}

For the saturation measurements and autocorrelation measurements of single emitters in the cavity (Fig. 3), we excite through the cavity fiber with light at a wavelength of $532 \mathrm{~nm}$. We calculate the excitation intensity $I$ inside the cavity from the measured transmitted power $P_{t}$, the outcoupling-mirror transmission $T_{1}$, and the evaluated excitation-mode waist $w_{e}$ according to $I=\left[8 P_{t} /\left(\pi w_{e}^{2} T_{1}\right)\right]$.

\section{Detection efficiency}

We infer the detection efficiency of the setup by coupling a laser at $690 \mathrm{~nm}$ into the cavity fiber with the cavity on resonance and measure the transmission of the mode coupled out of the cavity through the optics up to the APDs. A fraction of $67 \%$ of the light reaches the detectors at a cavity length of $1 \mu \mathrm{m}$. Together with the quantum efficiency of the APDs of 65\%, a detection efficiency of $43 \%$ at $690 \mathrm{~nm}$ is achieved.

\section{FDTD simulations}

We perform three-dimensional FDTD simulations with a commercial software (Lumerical). The diamond is modeled as a cube with a refractive index of 2.4 and different edge lengths. The individual mirror layers are implemented with parameters as realized in the experiment. A dipole source peaking at a wavelength of $690 \mathrm{~nm}$, with a Gaussian spectrum with a $1 / e$ width of $100 \mathrm{~nm}$, is placed at the center of the cube. The spectrum of the NV center is then accounted for in a second step by weighting the results with a Gaussian spectrum $S(\lambda)$ centered at $690 \mathrm{~nm}$, with a FWHM of $90 \mathrm{~nm}$. We perform simulations with a dipole orientation parallel and normal to the plane of the mirrors for different mirror separations.

\section{Quantum efficiency and metal mirror-induced nonradiative decay}

The quantum efficiency is defined via $\mathrm{QE}=\gamma_{\mathrm{r}} /$ $\left(\gamma_{\mathrm{r}}+\gamma_{\mathrm{nr}}\right)$, with the radiative-decay rate $\gamma_{\mathrm{r}}$ and the nonradiative rate $\gamma_{\mathrm{nr}}$, which together determine the excited state decay rate $1 / \tau_{0}=\gamma_{0}=\gamma_{\mathrm{r}}+\gamma_{\mathrm{nr}}$. While the intrinsic quantum efficiency of the studied emitters could, in principle, be inferred from a comparison of simulated and measured lifetime changes, the unknown contribution of other imperfections would lead to a large uncertainty. We note that an average value of $\mathrm{QE}=0.7$ was found in Ref. [42] for nanodiamonds.

The nonradiative-decay rate in the cavity $\gamma_{\mathrm{nr}}^{c}$ includes a possible contribution due to the presence of the nearby metal mirrors. To study the influence of the mirror, we simulate with FDTD the quantum efficiency of a dipoleoriented parallel to the mirror surface in a diamond cube (edge length, $30 \mathrm{~nm}$ ) on a silver mirror (layer thickness, $33 \mathrm{~nm}$ ), with an intermediate glass spacer layer of variable thickness. For glass layers of $20 \mathrm{~nm}$ thickness, the quantum efficiency amounts to $83 \%$ and, for layers thicker than $30 \mathrm{~nm}$, it exceeds $90 \%$. With applied spacer layers of $20 \mathrm{~nm}$ in Sec. III and $60 \mathrm{~nm}$ in Sec. IV and the additional separation between the dipole and the mirror due to the larger size of the nanodiamonds, we expect the amount of nonradiative decay to be less than $10 \%$, and we assume $\gamma_{\mathrm{nr}}^{c}=\gamma_{\mathrm{nr}}$ in the evaluation.

The Purcell effect can increase the quantum efficiency in the cavity, $\mathrm{QE}^{c}=(C+1) \gamma_{\mathrm{r}} /\left[(C+1) \gamma_{\mathrm{r}}+\gamma_{\mathrm{nr}}^{c}\right]$, while a finite quantum efficiency reduces the lifetime modification by the cavity, $\tau_{0} / \tau_{c}=\mathrm{QE} \cdot(C+1)$. The free-space and cavity-saturation count rates depend on the radiative rates $\gamma_{\mathrm{r}}$ and $C \gamma_{\mathrm{r}}$, respectively, which are independent of QE.

[1] Christian Kurtsiefer, Sonja Mayer, Patrick Zarda, and Harald Weinfurter, Stable Solid-State Source of Single Photons, Phys. Rev. Lett. 85, 290 (2000).

[2] I. Aharonovich, S. Castelletto, D. A. Simpson, C.-H. Su, A. D. Greentree, and S. Prawer, Diamond-based singlephoton emitters, Rep. Prog. Phys. 74, 076501 (2011).

[3] P. Neumann, R. Kolesov, B. Naydenov, J. Beck, F. Rempp, M. Steiner, V. Jacques, G. Balasubramanian, M. L. Markham, D. J. Twitchen, S. Pezzagna, J. Meijer, J. Twamley, F. Jelezko, and J. Wrachtrup, Quantum register based on coupled electron spins in a room-temperature solid, Nat. Phys. 6, 249 (2010).

[4] G. D. Fuchs, G. Burkard, P. V. Klimov, and D. D. Awschalom, A quantum memory intrinsic to single nitrogen-vacancy centres in diamond, Nat. Phys. 7, 789 (2011).

[5] P. C. Maurer, G. Kucsko, C. Latta, L. Jiang, N. Y. Yao, S. D. Bennett, F. Pastawski, D. Hunger, N. Chisholm, M. Markham et al., Room-temperature quantum bit memory exceeding one second, Science 336, 1283 (2012).

[6] Gopalakrishnan Balasubramanian, I. Y. Chan, Roman Kolesov, Mohannad Al-Hmoud, Julia Tisler, Chang Shin, Changdong Kim, Aleksander Wojcik, Philip R. Hemmer, Anke Krueger, Tobias Hanke, Alfred Leitenstorfer, Rudolf Bratschitsch, Fedor Jelezko, and Jörg Wrachtrup, Nanoscale imaging magnetometry with diamond spins under ambient conditions, Nature (London) 455, 648 (2008).

[7] J. R. Maze, P. L. Stanwix, J. S. Hodges, S. Hong, J. M. Taylor, P. Cappellaro, L. Jiang, M. V. Gurudev Dutt, E. Togan, A. S. Zibrov, A. Yacoby, R. L. Walsworth, and M. Lukin, Nanoscale magnetic sensing with an individual electronic spin in diamond, Nature (London) 455, 644 (2008). 
[8] P. Maletinsky, S. Hong, M. S. Grinolds, B. Hausmann, M. D. Lukin, R. L. Walsworth, M. Loncar, and A. Yacoby, A robust scanning diamond sensor for nanoscale imaging with single nitrogen-vacancy centres, Nat. Nanotechnol. 7, 320 (2012).

[9] Kerry J. Vahala, Optical microcavities, Nature (London) 424, 839 (2003).

[10] A. Auffèves, D. Gerace, J.-M. Gérard, M. França Santos, L. C. Andreani, and J.-P. Poizat, Controlling the dynamics of a coupled atom-cavity system by pure dephasing, Phys. Rev. B 81, 245419 (2010).

[11] A. Meldrum, P. Bianucci, and F. Marsiglio, Modification of ensemble emission rates and luminescence spectra for inhomogeneously broadened distributions of quantum dots coupled to optical microcavities, Opt. Express 18, 10230 (2010).

[12] Janik Wolters, Andreas W. Schell, Günter Kewes, Nils Nüsse, Max Schoengen, Henning Döscher, Thomas Hannappel, Bernd Löchel, Michael Barth, and Oliver Benson, Enhancement of the zero phonon line emission from a single nitrogen vacancy center in a nanodiamond via coupling to a photonic crystal cavity, Appl. Phys. Lett. 97, 141108 (2010).

[13] Dirk Englund, Brendan Shields, Kelley Rivoire, Fariba Hatami, Jelena Vucovic, Hongkun Park, and Mikhail D. Lukin, Deterministic coupling of a single nitrogen vacancy center to a photonic crystal cavity, Nano Lett. 10, 3922 (2010).

[14] Paul E. Barclay, Kai-Mei C. Fu, Charles Santori, Andrei Faraon, and Raymond G. Beausoleil, Hybrid Nanocavity Resonant Enhancement of Color Center Emission in Diamond, Phys. Rev. X 1, 011007 (2011).

[15] A. Faraon, C. Santori, Z. Huang, V. M. Acosta, and R. G. Beausoleil, Coupling of Nitrogen-Vacancy Centers to Photonic Crystal Cavities in Monocrystalline Diamond, Phys. Rev. Lett. 109, 033604 (2012).

[16] T. van der Sar, J. Hagemeier, W. Pfaff, E. C. Heeres, S. M. Thon, H. Kim, P. M. Petroff, T. H. Oosterkamp, D. Bouwmeester, and R. Hanson, Deterministic nanoassembly of a coupled quantum emitter-photonic crystal cavity system, Appl. Phys. Lett. 98, 193103 (2011).

[17] B. J. M. Hausmann, B. J. Shields, Q. Quan, Y. Chu, N. P. de Leon, R. Evans, M. J. Burek, A. S. Zibrov, M. Markham, D. J. Twitchen et al., Coupling of NV centers to photonic crystal nanobeams in diamond, Nano Lett. 13, 5791 (2013).

[18] Jonathan C. Lee, David O. Bracher, Shanying Cui, Kenichi Ohno, Claire A. McLellan, Xingyu Zhang, Paolo Andrich, Benjamin Aleman, Kasey J. Russell, Andrew P. Magyar et al., Deterministic coupling of delta-doped nitrogen vacancy centers to a nanobeam photonic crystal cavity, Appl. Phys. Lett. 105, 261101 (2014).

[19] Luozhou Li, Tim Schröder, Edward H. Chen, Michael Walsh, Igal Bayn, Jordan Goldstein, Ophir Gaathon, Matthew E. Trusheim, Ming Lu, Jacob Mower et al., Coherent spin control of a nanocavity-enhanced qubit in diamond, Nat. Commun. 6, 6173 (2015).

[20] Janine Riedrich-Möller, Sebastien Pezzagna, Jan Meijer, Christoph Pauly, Frank Mücklich, Matthew Markham, Andrew M. Edmonds, and Christoph Becher, Nanoimplantation and Purcell enhancement of single nitrogen-vacancy centers in photonic crystal cavities in diamond, Appl. Phys. Lett. 106, 221103 (2015).

[21] Tim Schröder, Sara L. Mouradian, Jiabao Zheng, Matthew E. Trusheim, Michael Walsh, Edward H. Chen, Luozhou Li, Igal Bayn, and Dirk Englund, Quantum nanophotonics in diamond, J. Opt. Soc. Am. B 33, B65 (2016).

[22] Roland Albrecht, Alexander Bommer, Christian Deutsch, Jakob Reichel, and Christoph Becher, Coupling of a Single Nitrogen-Vacancy Center in Diamond to a Fiber-Based Microcavity, Phys. Rev. Lett. 110, 243602 (2013).

[23] Hanno Kaupp, Christian Deutsch, Huan-Cheng Chang, Jakob Reichel, Theodor W. Hänsch, and David Hunger, Scaling laws of the cavity enhancement for nitrogenvacancy centers in diamond, Phys. Rev. A 88, 053812 (2013).

[24] Roland Albrecht, Alexander Bommer, Christoph Pauly, Frank Mücklich, Andreas W. Schell, Philip Engel, Tim Schröder, Oliver Benson, Jakob Reichel, and Christoph Becher, Narrow-band single photon emission at room temperature based on a single nitrogen-vacancy center coupled to an all-fiber-cavity, Appl. Phys. Lett. 105, 073113 (2014).

[25] E. Janitz, M. Ruf, M. Dimock, A. Bourassa, J. Sankey, and L. Childress, Fabry-perot microcavity for diamond-based photonics, Phys. Rev. A 92, 043844 (2015).

[26] S. Johnson, P. R. Dolan, T. Grange, A. A. P. Trichet, G. Hornecker, Y. C. Chen, L. Weng, G. M. Hughes, A. A. R. Watt, A. Auffeves, and J. M. Smith, Tunable cavity coupling of the zero phonon line of a nitrogen-vacancy defect in diamond, New J. Phys. 17, 122003 (2015).

[27] H. S. Knowles, D. M. Kara, and M. Atatüre, Observing bulk diamond spin coherence in high-purity nanodiamonds, Nat. Mater. 13, 21 (2013).

[28] Hrishikesh Kelkar, Daqing Wang, Diego Martin-Cano, Björn Hoffmann, Silke Christiansen, Stephan Götzinger, and Vahid Sandoghdar, Sensing Nanoparticles with a Cantilever-Based Scannable Optical Cavity of Low Finesse and Sub- $\lambda^{3}$ Volume, Phys. Rev. Applied 4, 054010 (2015).

[29] D. Hunger, T. Steinmetz, Y. Colombe, C. Deutsch, T. W. Hänsch, and J. Reichel, A fiber Fabry-Perot cavity with high finesse, New J. Phys. 12, 065038 (2010).

[30] C. Toninelli, Y. Delley, T. Stöferle, A. Renn, S. Götzinger, and V. Sandoghdar, A scanning microcavity for in situ control of single-molecule emission, Appl. Phys. Lett. 97, 021107 (2010).

[31] A. Muller, E. B. Flagg, J. R. Lawall, and G. S. Solomon, Ultrahigh-finesse, low-mode-volume Fabry-Perot microcavity, Opt. Lett. 35, 2293 (2010).

[32] Philip R. Dolan, Gareth M. Hughes, Fabio Grazioso, Brian R. Patton, and Jason M. Smith, Femtoliter tunable optical cavity arrays, Opt. Lett. 35, 3556 (2010).

[33] M. Trupke, E. A. Hinds, S. Eriksson, E. A. Curtis, Z. Moktadir, E. Kukharenka, and M. Kraft, Microfabricated high-finesse optical cavity with open access and small volume, Appl. Phys. Lett. 87, 211106 (2005).

[34] Russell J. Barbour, Paul A. Dalgarno, Arran Curran, Kris M. Nowak, Howard J. Baker, Denis R. Hall, Nick G. Stoltz, Pierre M. Petroff, and Richard J. Warburton, A tunable microcavity, J. Appl. Phys. 110, 053107 (2011). 
[35] Matthias Mader, Jakob Reichel, Theodor W. Hänsch, and David Hunger, A scanning cavity microscope, Nat. Commun. 6, 7249 (2015).

[36] Thomas Hümmer, Jonathan Noe, Matthias S. Hofmann, Theodor W. Hänsch, Alexander Högele, and David Hunger, Cavity-enhanced raman microscopy of individual carbon nanotubes, Nat. Commun. 7, 12155 (2016).

[37] D. Hunger, C. Deutsch, R. J. Barbour, R. J. Warburton, and J. Reichel, Laser micro-fabrication of concave, lowroughness features in silica, AIP Adv. 2, 012119 (2012).

[38] Konstantin Ott, Sebastien Garcia, Ralf Kohlhaas, Klemens Schüppert, Peter Rosenbusch, Romain Long, and Jakob Reichel, Millimeter-long fiber Fabry-Perot cavities, Opt. Express 24, 9839 (2016).

[39] For some geometries, it is advantageous to create the concave profile before tip shaping.

[40] M. Steiner, F. Schleifenbaum, C. Stupperich, A. V. Failla, A. Hartschuh, and A. J. Meixner, A new microcavity design for single molecule detection, J. Lumin. 119-120, 167 (2006).

[41] M. Steiner, A. Hartschuh, R. Korlacki, and A. J. Meixner, Highly efficient, tunable single photon source based on single molecules, Appl. Phys. Lett. 90, 183122 (2007).

[42] F. A. Inam, M. D. W. Grogan, M. Rollings, T. Gaebel, J. M. Say, C. Bradac, T. A. Birks, W. J. Wadsworth, S. Castelletto, J. R. Rabeau, and M. J. Steel, Emission and nonradiative decay of nanodiamond NV centers in a low refractive index environment, ACS Nano 7, 3833 (2013).

[43] W. Lukosz and R. E. Kunz, Light emission by magnetic and electric dipoles close to a plane interface. I. Total radiated power, J. Opt. Soc. Am. 67, 1607 (1977).

[44] W. Lukosz, Light emission by magnetic and electric dipoles close to a plane dielectric interface. III. Radiation patterns of dipoles with arbitrary orientation, J. Opt. Soc. Am. 69, 1495 (1979).

[45] E. A. Hinds, Cavity Quantum Electrodynamics, in Advances in Atomic, Molecular, and Optical Physics, Vol. 28, edited by D. Bates and B. Bederson (Academic Press, 1990), pp. 237-289.

[46] Ziyun Di, Helene V. Jones, Philip R. Dolan, Simon M. Fairclough, Matthew B. Wincott, Johnny Fill, Gareth M. Hughes, and Jason M. Smith, Controlling the emission from semiconductor quantum dots using ultra-small tunable optical microcavities, New J. Phys. 14, 103048 (2012).

[47] As long as no collective effects become relevant (which is the case here), the behavior of the ensemble is not expected to differ from that of single emitters.

[48] Nitin Mohan, Yan-Kai Tzeng, Liling Yang, Yi-Ying Chen, Yuen Yung Hui, Chia-Yi Fang, and Huan-Cheng Chang, Sub-20-nm fluorescent nanodiamonds as photostable biolabels and fluorescence resonance energy transfer donors, Adv. Mater. 22, 843 (2010).
[49] Shu-Jung Yu, Ming-Wei Kang, Huan-Cheng Chang, Kuan-Ming Chen, and Yueh-Chung Yu, Bright fluorescent nanodiamonds: No photobleaching and low cytotoxicity, J. Am. Chem. Soc. 127, 17604 (2005).

[50] Alexey I. Chizhik, Anna M. Chizhik, Dmitry Khoptyar, Sebastian Bär, Alfred J. Meixner, and Jörg Enderlein, Probing the radiative transition of single molecules with a tunable microresonator, Nano Lett. 11, 1700 (2011).

[51] In the measurement shown in Fig. 4(e), we observe a deviation of the actual cavity length from the expected value below $d \sim 500 \mathrm{~nm}$, probably due to the cavity fiber touching the large mirror with one of its edges. We have adjusted the distance calibration below $500 \mathrm{~nm}$ to restrict the cavity lengths to $d>100 \mathrm{~nm}$.

[52] We note that, in general, a multiexponential decay is expected due to the position and dipole-orientation distribution of the color centers in the nanodiamond. Fitting the data with stretched exponentials [M. N. Berberan-Santos, E. N. Bodunov, and B. Valeur, Mathematical functions for the analysis of luminescence decays with underlying distributions 1. Kohlrausch decay function (stretched exponential), Chem. Phys. 315, 171 (2005)] yields a larger lifetime modulation, such that the evaluation shown is a conservative estimate.

[53] Thomas M. Babinec, Birgit J. M. Hausmann, Mughees Khan, Yinan Zhang, Jeronimo R. Maze, Philip R. Hemmer, and Marko Loncar, A diamond nanowire single-photon source, Nat. Nanotechnol. 5, 195 (2010).

[54] We note that a small amount of parasitic lifetime modulation seems to be also present, again probably due to the varying amount of excitation light coupled into the cavity and the corresponding variation of the background contribution. It can be seen at a large mirror separation (e.g., for $d>1.3 \mu \mathrm{m}$ ) with a periodicity of $270 \mathrm{~nm}$.

[55] S. Ali Momenzadeh, Rainer J. Stöhr, Felipe Favaro de Oliveira, Andreas Brunner, Andrej Denisenko, Sen Yang, Friedemann Reinhard, and Jörg Wrachtrup, Nanoengineered diamond waveguide as a robust bright platform for nanomagnetometry using shallow nitrogen vacancy centers, Nano Lett. 15, 165 (2015).

[56] Carsten Arend, Patrick Appel, Jonas Nils Becker, Marcel Schmidt, Martin Fischer, Stefan Gsell, Matthias Schreck, Christoph Becher, Patrick Maletinsky, and Elke Neu, Site selective growth of heteroepitaxial diamond nanoislands containing single SiV centers, Appl. Phys. Lett. 108, 063111 (2016).

[57] See http://www.fp7wasps.org.

[58] Sh. A. Furman and A. V. Tikhonravov, Basics of Optics of Multilayer Systems (World Scientific, Singapore, 1992).

[59] H. Becker, S. E. Burns, N. Tessler, and R. H. Friend, Role of optical properties of metallic mirrors in microcavity structures, J. Appl. Phys. 81, 2825 (1997). 